\title{
Determination of Parameters of Surface Acoustic Wave Vibration Sensors with the Help of Gravitational Field
}

\author{
J. FilipiaK* AND G. STECZKO \\ Institute of Electronic and Control Systems, Technical University of Częstochowa \\ Armii Krajowej 17, 42-200 Częstochowa, Poland
}

\begin{abstract}
This work presents results of experimental tests of surface acoustic wave vibration sensor. Measurements of the static sensitivity, resonance frequency, frequency amplitude characteristics and linearity were carried out. Measurements were carried out with the help of the acceleration of gravity and a digital oscilloscope.
\end{abstract}

DOI: 10.12693 /APhysPolA.124.410

PACS: 77.65.Dq, 68.35.Iv

\section{Introduction}

The structure of probes for measurement of mechanical vibrations bases on three basic types of vibration sensors: seismometers, geophones, accelerometers [1-4]. A measuring sensor should remain in a rigid contact with the substrate. It transforms substrate vibrations into an output electric signal proportional to the vibration parameters. For harmonic vibrations the substrate displacement is determined by the following relation: $Y(t)=D \sin \omega t$. The vibration parameters are as follows: the vibration frequency $\omega$ and one of the amplitudes: the displacement amplitude $D$, the vibration velocity amplitude $[(\mathrm{d} Y(t) / \mathrm{d} t)=D \omega \cos \omega t]$ or the vibration acceleration amplitude $\left[\left(\mathrm{d}^{2} Y(t) / \mathrm{d} t^{2}\right)=-D \omega^{2} \sin \omega t\right]$.

The output signal from a seismometer and a geophone is approximately proportional to the enforced vibration velocity, where the output signal from an accelerometer is approximately proportional to the enforced vibration acceleration.

\section{Design and parameters of surface acoustic wave vibration sensor}

Measurements of mechanical vibration parameters can be performed using a surface acoustic wave vibration sensor (SAW-VS) [5-7]. A SAW vibration sensor is shown in Fig. 1. The main part is an anisotropic plate made of a piezoelectric material. One side of the plate is stiffly attached to the sensor casing, while the other free side is loaded with the seismic mass. On the upper surface of the plate a SAW delay line in the form of a four-terminal network has been made. Any motion of the casing of a sensor creates vibrations of the plate and an alternation in the time delay of a SAW delay line. It results in a high frequency signal phase which goes through such a line. The magnitude of an alternation of signal phase is proportional to the alternation in time delay of the SAW delay line.

*corresponding author; e-mail: gem@gemsc.com.pl

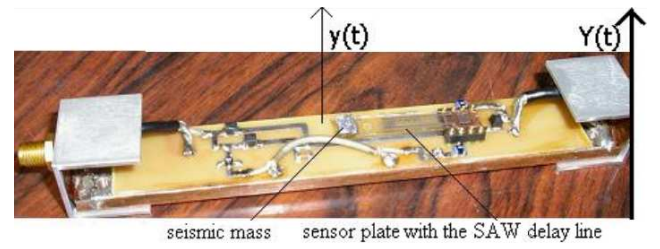

Fig. 1. Surface acoustic wave vibration sensor.

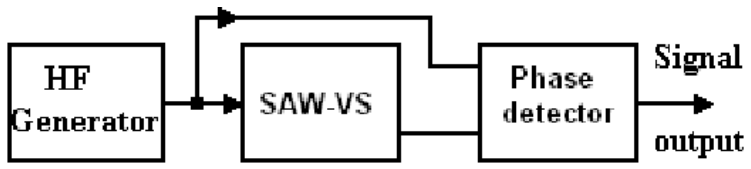

Fig. 2. Measuring system of SAW vibration sensor.

A measuring probe system with SAW-VS is shown in Fig. 2. It consists of a measuring signal generator (HF Generator), a sensor (SAW-VS) and a phase detector with a system of filters. Measuring probe output signal is a voltage and its value is proportional to the phase shift of the measuring signal passing through the SAW vibration sensor.

The value of the line delay is proportional to the motion of the upper side of the plate described by the $y(t)$ function. Basic parameters of the sensor are its sensitivity, resonance characteristic, and linearity. These parameters depend on the plate structure, the structure of the SAW delay line and on the probe electronic measuring system. Using models of the mentioned components one can make a measuring probe having specified parameters. To describe movement parameters of an anisotropic, piezoelectric plate of a sensor a model with one degree of freedom was formulated [8]. The resonance frequency of the sensor plate $\omega$ is given by the relation

$$
\omega_{\mathrm{r}}=\omega_{0} \sqrt{1-\frac{\omega_{0}^{2} \tau^{2}}{4}},
$$

where

$$
\omega_{0}=3.5172\left(\frac{h}{l^{2}}\right) \sqrt{\frac{E_{\mathrm{e}}}{12 \rho}} \sqrt{\frac{1}{1+r 3.9689}} .
$$

$\rho$ - plate density, $h$ - plate thickness, $l$ - plate length, 
$r$ is the relation of the seismic mass to the mass of the sensor plate, $E_{\mathrm{e}}$ - equivalent Young's modulus, $\tau-$ equivalent material damping coefficient.

The movement of the plate end is given by the function

$$
\begin{aligned}
y(t) & =A \exp \left(-\frac{\omega_{0}^{2} \tau t}{2}\right) \sin \left(\omega_{\mathrm{r}}(1+\varphi)\right)-\frac{4 \omega_{\mathrm{r}}}{4-\omega_{0}^{2} \tau^{2}} \\
\times & \int_{0}^{t}\left(\tau \frac{\mathrm{d} Y(\xi)}{\mathrm{d} t}+Y(\xi)\right) \exp \left(-\frac{\omega_{0}^{2} \tau}{2}(t-\xi)\right) \\
& \times \sin \left(\omega_{\mathrm{r}}(t-\xi)\right) \mathrm{d} \xi,
\end{aligned}
$$

where the constants $A$ and $\varphi$ are calculated from the initial conditions.

Both components of the solution comprise the function

$$
\varphi_{\delta}(t)=A \mathrm{e}^{-\frac{\omega_{0}^{2} \tau}{2} t} \sin \left(\omega_{\mathrm{r}} t+\varphi\right) .
$$

This is the system impulse response to the sensor excitation by the $\delta$-Dirac impulse.

The frequency characteristic of the sensor plate is associated with the impulse response by the Fourier transform. The form of the theoretical amplitude-frequency characteristic of the SAW vibration sensor is as follows [8]:

$$
H(\omega)=\sqrt{\frac{1+(\omega \tau)^{2}}{\left[1-\left(\omega / \omega_{\mathrm{r}}\right)^{2}\right]^{2}+(\omega \tau)^{2}}} .
$$

An exemplary theoretical amplitude frequency characteristic for the SAW vibration sensor of a resonance frequency of $29 \mathrm{~Hz}$ is shown in Fig. 3.

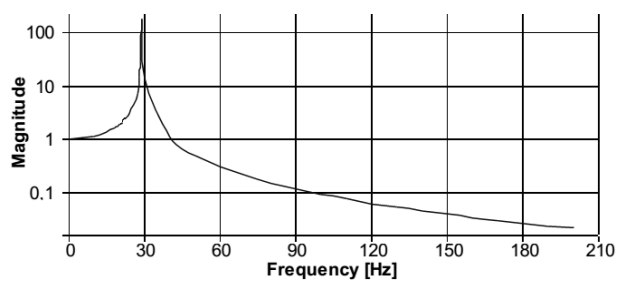

Fig. 3. Theoretical amplitude-frequency response of the plate.

For the purpose of tests a vibration sensor with SAW was prepared, and the plate was made of the ST quartz $0.5 \mathrm{~mm}$ thick. The equivalent Young modulus for the ST quartz is $76 \mathrm{GPa}$ and the equivalent material damping coefficient is $29.3 \mu \mathrm{s}$ [8]. The frequency of the measuring signal was $74 \mathrm{MHz}$.

\section{Experimental testing of the sensor parameters}

The basic parameters of the SAW vibration sensor were performed with a probe measuring system presented in Fig. 2. The sensor output signal was recorded with a digital oscilloscope type DSO-X 2024A operating in the refresh mode (Roll).

Experimental impulse responses of a sensor with a $57 \mathrm{~mm}$ long plate without a seismic mass and after placing the seismic mass of $1.8 \mathrm{~g}$ on the sensor plate are presented in Fig. 4. These responses were generated by an impulse hitting on the sensor casing. The resonance frequency of the sensor was measured automatically during recording of the impulse response on the oscilloscope. The resonance frequency of the sensor without the seismic mass was $81 \mathrm{~Hz}$ and for the plate with the mass of $1.8 \mathrm{~g}$ was down to $29 \mathrm{~Hz}$. The obtained results of experimental research comply with the results obtained from the theoretical model of the sensor plate.

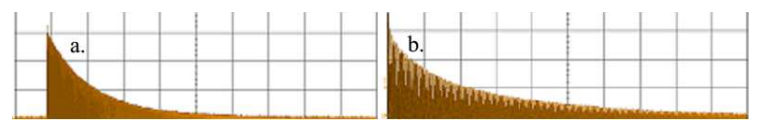

Fig. 4. Sensor impulse response of a sensor with a $57 \mathrm{~mm}$ long plate without the seismic mass (a) and with the $1.8 \mathrm{~g}$ seismic mass $(\mathrm{b})$.

A direct measurement of frequency characteristics of a sensor requires proper test stations. Knowing their relation with the impulse response one can obtain information on their shape. Figure 5 shows the Fourier transform of the sensor impulse responses from Fig. 4. They were calculated with the help of the VEE Agilent programme operating in combination with an oscilloscope. Placing the seismic mass causes the resonance frequency to shift and practically a decay of the harmonic (its level is $-40 \mathrm{~dB}$ for a plate without a seismic mass). Obtained results confirm the accuracy of the used model.

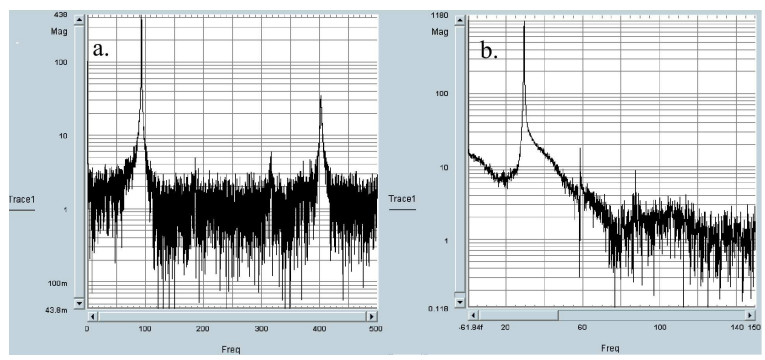

Fig. 5. Spectrum of impulse responses of sensor shown in Fig. 4, plate without seismic mass (a) and with $1.8 \mathrm{~g}$ seismic mass (b).

The sensitivity is one of basic parameters. It is the relation of a change of the level of the output signal from a sensor " $\Delta U_{\text {out }}$ " to the change of the value of the acceleration acting on the " $\Delta g$ " sensor. To determine it we must be able to change the value of the acceleration acting on the sensor. Figure 6 presents the way the acceleration value changes by changing the sensor position in relation to the gravitational acceleration direction.
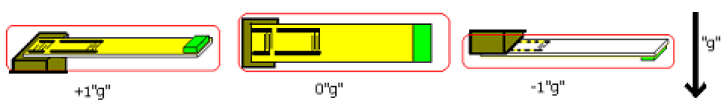

Fig. 6. Determination of static sensitivity of sensor. 


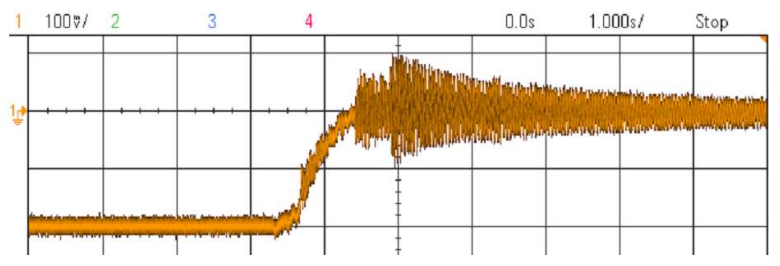

Fig. 7. Method of determination of sensor static sensitivity.

Figure 7 presents an experimentally determined change of the sensor output signal by changing by " $2 g$ " the acceleration acting on it.

The sensor sensitivity was determined from the relation

$$
S=\frac{\Delta U_{\text {out }}}{\Delta g}=\frac{U_{\text {out },+1 g}-U_{\text {out },-1 g}}{2 g} .
$$

The determined sensor sensitivity is $100 \mathrm{mV} / \mathrm{g}$.

Much more difficult is to determine the sensor linearity. It requires to determine the dependence of the " $\Delta U_{\text {out } \text { " }}$ output signal on the value of the acceleration acting on the sensor " $\Delta g$ ". Figure 6 shows the way to obtain three acceleration values ( $+1^{\prime \prime} g$ ", 0 " $g$ ", -1 " $g$ ") acting on the sensor. Basing on that it was attempted to estimate the sensor linearity in the manner presented in Fig. 8.

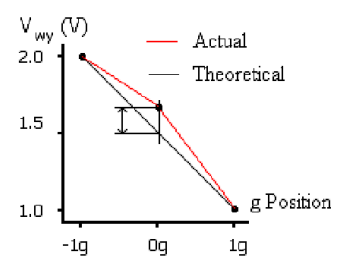

Fig. 8. The way to determine the sensor linearity.

The relation below is the linearity measure

$$
\mathrm{ML}=U_{\text {out }, 0 g}-0.5\left(U_{\text {out },+1 g}+U_{\text {out },-1 g}\right) \text {. }
$$

During performed experimental research the value of $\mathrm{ML} \approx 0$ was obtained thus allowing to confirm a good static linearity of a SAW vibration sensor within changes of the static linearity by " $2 g$ ". This simple method allows only to evaluate the sensor linearity but it is used for all the types of acceleration sensors.

\section{Conclusions}

This work presents results of the experimental research of surface acoustic wave vibration sensors. Measurements of the static sensitivity, the resonance frequency, the impulse response and the linearity were made. Obtained results allowed to confirm the appropriateness of the sensors modeling and determining their basic parameters. The research was conducted using very simple methods using only the gravitational acceleration and a digital oscilloscope.

The impulse response of the surface acoustic wave vibration sensors of a resonance frequency of $91 \mathrm{~Hz}$ and its spectrum is presented in Fig. 9. A digital oscilloscope is practically the only instrument required to examine static parameters of vibration sensors within the presented range.

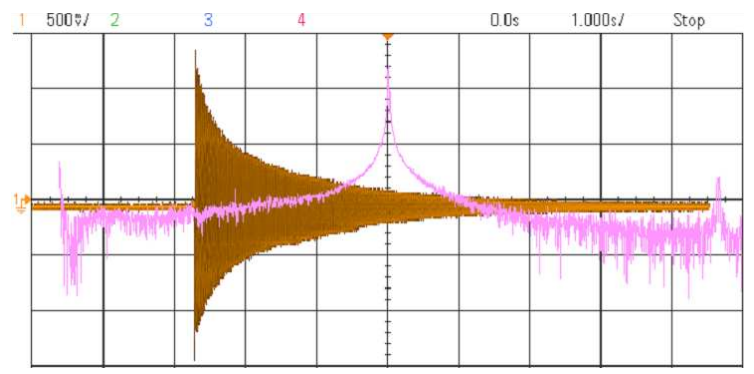

Fig. 9. Impulse response of surface acoustic wave vibration sensor (yellow) and its spectrum (pink).

Taking the advantage of the possibility to measure the sensor output signal as a function of its rotation in the earth gravitational field will make it possible to determine the static thru-sensitivity and next a precise measurement of the linearity within the change of the constant acceleration by " $2 g$ ". Determination of the dynamic sensitivity, the frequency-amplitude characteristic and the dynamic linearity requires to carry out tests on special test/research stations. The results of these tests can be interesting since the sensor resonance frequency can be selected over a very wide range: from a few $\mathrm{Hz}$ to several $\mathrm{kHz}$ and at very small resonance frequencies occurs a multiple increase of the free vibration amplitude. The market offer does not include surface acoustic wave vibration sensors. Their properties we have mentioned above and possible measurements of constant accelerations offer an alternative solution to use these sensors in lieu of currently used vibration sensors.

\section{References}

[1] J. Kornowski, I. Gołda, Górnictwo i Geologia 6.3, 87 (2011) (in Polish).

[2] Piezoelectric Accelerometer and Vibration Preamplifier Handbook, Bruel and Kjaer, Naerum, Denmark 1978.

[3] I. Lee, G.H. Yoon, J. Park, S. Seok, K. Chun, K. Lee, Sensors Actuators A 119,8 (2005).

[4] wWw.sensonics.co.uk, ww.metrozet.com, wWw. hansfordsensors.pl, ww.reftek.com, www.EQMet. com, www.metrxvibration.com.

[5] J. Filipiak, C. Kopycki, Sensors Actuators 76, 318 (1999).

[6] W. Jakubik, M. Urbanczyk, E. Maciak, T. Pustelny, Bull. Pol. Acad. Sci. Techn. Sci. 56, 133 (2008).

[7] J. Filipiak, L. Solarz, G. Steczko, Sensors 11, 11809 (2011).

[8] J. Filipiak, Surface Acoustic Wave Acceleration Sensors, Technical University of Częstochowa, Częstochowa 2006 (in Polish). 